\title{
Thermal properties, curing characteristics and water absorption of soybean oil-based thermoset
}

\author{
S. G. Tan, W. S. Chow ${ }^{*}$ \\ School of Materials and Mineral Resources Engineering, Engineering Campus, Universiti Sains Malaysia, Nibong Tebal \\ 14300 Penang, Malaysia
}

Received 20 October 2010; accepted in revised form 10 December 2010

\begin{abstract}
Epoxidized soybean oil (ESO) was successfully thermal-cured by using methylhexahydrophthalic anhydride (MHHPA) curing agent, in the presence of tetraethylammonium bromide (TEAB) catalyst of varied concentration $(0.3-0.8$ phr). The polyesterification process of ESO thermoset was proven and supported by Fourier transforms infrared spectroscopy (FTIR) and gas chromatography-mass spectroscopy analysis (GC-MS). A possible chemical reaction of the MHHPA, TEAB and ESO was proposed based on the experimental work. Differential scanning calorimetry (DSC) and dynamic mechanical analysis (DMA) revealed that there is a positive relationship between the degree of conversion and crosslink density of ESO thermoset with TEAB concentration. The kinetics of water absorption of the ESO thermoset were found to conform to Fickian law behavior.
\end{abstract}

Keywords: biopolymers, epoxidized soybean oil, thermosetting resin, thermal properties, water absorption

\section{Introduction}

Traditionally, epoxidized vegetable oils (EVO) are used as poly(vinyl chloride) plasticizers, stabilizers and lubricants. Recently, modified vegetable oils could be used to synthesize segmented polyurethane [1], thermoplastic polyurethanes [2], poly (methyl methacrylate)-multigraft copolymers [3], novel thermosets and rubbers [4-5] and 'green' composites [6]. In recent years, there has been substantial growth in biopolymers from EVO. These EVO from biological origin substrates could bring along numerous advantages and new beneficial properties, which may not be able to gain from petroleum-based epoxy resins. For example, EVO have been evaluated as ecological and environmental friendly alternative for petroleum-based epoxy resins since they are neutral in the carbon dioxide cycle and are readily biodegradable. Other advantages of

\footnotetext{
${ }^{*}$ Corresponding author, e-mail: chowwenshyang@yahoo.com (c) BME-PT
}

the EVO include cost effectiveness, renewability and availability.

In general, epoxidized soybean oil (ESO) is a triglyceride made up of a complex multi-component mixture of functionalized oleic, linoleic and linolenic acid methyl esters as well as saturated fatty acids (i.e. palmitic and stearic acids). It has been recognized in the literature that these functionalized fatty acids in ESO could be thermally cured with a thermal latent initiator [7], thermally cured with acid anhydride under the catalytic reaction of tertiary amines catalyst [8] and ultraviolet (UV) cured in the presence of photo-initiators [9]. However, saturated fatty acids in ESO do not take part in the polymer network formation. This is due to the fact that they do not possess any epoxy functional groups in their saturated backbone structures.

Specifically, in the open literature, there exist numerous models to explain the curing mechanisms for 
ESO. Jin and Park [7] proposed thermally induced ring opening polymerization route for ESO thermoset initiated by N-benzylquinoxalinium hexafluoroantimonate thermal latent initiator. Ortiz et al. [9] reported that radical induced cationic photopolymerization of ESO thermoset in the presence of diarylinodonium salt photo-initiator follows conventional cationic polymerization mechanism and chain reaction mechanism. Gao [10] proposed that the curing mechanism of catalytic ESO-anhydride thermosets involves reaction of the tertiary amine with ESO monomer followed by the ring opening of the anhydride functional group with the alkoxide.

Although the EVO based thermosetting materials are more environmental friendly, they tend to suffer from shortcomings in terms of long curing schedule, high curing temperature, poor thermo-physical properties and high degree of water uptake. In order to overcome some of these limitations, our work aims to chemically synthesize a thermally curable ESO thermosetting resin in the presence of the tetraethylammonium bromide (TEAB) catalyst. It is hypothesized that the TEAB-catalyzed ESO thermosetting resin can be prepared using shorter curing schedules and lower curing temperature due to the fast cure rate of the TEAB catalyst. Accordingly, the primary objective of this present study is to investigate the effect of TEAB catalyst concentration on the curing characteristics, thermal properties and kinetics of water absorption of ESO thermoset. A plausible curing mechanism of the thermal curable ESO thermoset under the catalytic reaction of TEAB catalyst will also be proposed.

\section{Experimental}

\subsection{Materials}

Epoxidized soybean oil (ESO; trade name Mingchen ESO-1) resin with $6.1 \mathrm{wt} \%$ epoxy oxirane content and molecular weight of about $950 \mathrm{~g} / \mathrm{mol}$ was purchased from Shangdong Longkou Longda Chemi-

Table 1. Materials designation and composition of ESO thermoset

\begin{tabular}{|c|c|}
\hline Materials designation & $\begin{array}{c}\text { Concentration of TEAB catalyst } \\
\text { [phr] }\end{array}$ \\
\hline ES_0.3A & 0.3 \\
\hline ES_0.4A & 0.4 \\
\hline ES_0.5A & 0.5 \\
\hline ES_0.6A & 0.6 \\
\hline ES_0.7A & 0.7 \\
\hline ES_0.8A & 0.8 \\
\hline
\end{tabular}

cal Industry Co., Ltd., China. Industrial grade methylhexahydropthalic anhydride (MHHPA) curing agent was purchased from CAPE Technology Sdn. Bhd., Malaysia. Tetraethylammonium bromide (TEAB: code T7012) catalyst was supplied by Sigma-Aldrich, Malaysia. The materials designation and composition of the ESO thermoset were summarized in Table 1.

\subsection{Preparation of thermal curable ESO thermoset}

MHHPA curing agent was pre-mixed with TEAB catalyst at a predetermined ratio. ESO resin and MHHPA/TEAB mixture were then mixed at room temperature and stirred mechanically. The mixture was then poured into the cavities of mould and subjected to thermal curing process in an oven at $140^{\circ} \mathrm{C}$ for 3 hours. The dimension of the mould used to prepare the ESO thermoset is $300 \mathrm{~mm} \times 100 \mathrm{~mm} \times 3 \mathrm{~mm}$ (length $\times$ width $\times$ thickness).

\subsection{Curing characteristics of ESO thermoset}

The curing characteristics of the TEAB-catalyzed ESO thermoset were characterized using Fourier transform infrared spectroscopy (Spectrum 100 FTIR, Perkin Elmer, USA), gas chromatographymass spectroscopy (GC-MS, Perkin Elmer Clarus 600T, USA), DSC Diamond Analyzer (Perkin Elmer, USA) and DMA 8000 (Perkin Elmer, USA).

The curing characteristics of ESO thermoset which thermally cured for 1, 2 and 3 hours were determined with FTIR. The FTIR spectra of the thermal curable ESO thermoset from the wavelength of 4000 to $550 \mathrm{~cm}^{-1}$ were recorded as the infrared radiation transmitted through the sample. The carbonyl index (C.I.) values of the samples were calculated based on the ratio of absorbance $(A)$ at two different wave-numbers as shown in Equation (1):

C.I. $=\frac{A_{1700}}{A_{1456}}$

where $A_{1700}$ represents the absorbance band at $1700 \mathrm{~cm}^{-1}$ due to the presence of carbonyl stretch of the aromatic acid whereas $A_{1456}$ represents the band at $1456 \mathrm{~cm}^{-1}$ corresponding to the absorbance from the presence of methyl group in MHHPA curing agent, which is taken as a reference band.

The pure ESO and extracted molecular fragments of the ESO thermoset were analyzed by gas chro- 
matography mass spectroscopy (GC-MS). One $\mu 1$ injection volume using splitless mode was performed on capillary column $(30 \mathrm{~m} \times 250 \mu \mathrm{m})$ with helium flows at constant pressure. The initial temperature, injection temperature and transfer temperature of the GC-MS test were set at 65,250 and $180^{\circ} \mathrm{C}$, respectively. The holding time was set for $10 \mathrm{~min}-$ utes at a ramp rate of $8^{\circ} \mathrm{C} / \mathrm{min}$. The molecular fragments of the ESO were determined using TurboMass $^{\mathrm{TM}}$ software.

The degree of conversion of ESO thermoset was determined using DSC and calculated based on Equation (2). The crosslink density $\left(v_{\mathrm{c}}\right)$ and molecular weight between cross-linking $\left(M_{\mathrm{c}}\right)$ of the ESO thermoset were determined using DMA and calculated based on Equations (3) and (4) respectively [11-12]:

$$
\begin{aligned}
& \alpha=\frac{\Delta H_{\mathrm{c}}-\Delta H_{\mathrm{r}}}{\Delta H_{\mathrm{c}}} \\
& v_{\mathrm{c}}=\frac{E^{\prime}}{3 R T} \\
& M_{\mathrm{c}}=\frac{d}{v_{\mathrm{c}}}
\end{aligned}
$$

where $\alpha$ is the degree of conversion, $\Delta H_{\mathrm{c}}$ is the total exothermic heat generated for a fully cured system and $\Delta H_{\mathrm{r}}$ is the total residual exothermic heat generated during a specified period of time. $v_{\mathrm{c}}$ is the crosslink density for the epoxy network, $E^{\prime}$ is the storage modulus of the thermoset in the rubbery plateau region at $T_{\mathrm{g}}+40^{\circ} \mathrm{C}, R$ is the gas constant, $T$ is the absolute temperature, $d$ is the density $\left(1.1 \mathrm{~g} / \mathrm{cm}^{3}\right)$ and $M_{\mathrm{c}}$ is the molecular weight between crosslink.

\subsection{Thermal characterization of ESO thermoset}

The curing profile of ESO thermoset was examined using DSC Diamond Analyzer (Perkin Elmer, USA). Approximately $20 \mathrm{mg}$ of the sample was placed into the DSC aluminium pans, followed by thermal scanning from 30 to $300^{\circ} \mathrm{C}$ at a heating rate of $10^{\circ} \mathrm{C} / \mathrm{min}$, in nitrogen gas atmosphere. The onset curing temperature $\left(T_{\text {onset }}\right)$, the temperature where maximum cross-linking reaction takes place $\left(T_{\text {peak }}\right)$ and the total heat released during curing reaction $(\Delta H)$ of ESO thermoset were determined. The dynamic mechanical properties of ESO thermoset were deter- mined using DMA 8000 (Perkin Elmer, USA). The specimen with the dimension of $25 \mathrm{~mm} \times 10 \mathrm{~mm} \times$ $2 \mathrm{~mm}$ was heated from -100 to $200^{\circ} \mathrm{C}$ at a heating rate of $2^{\circ} \mathrm{C} / \mathrm{min}$ in nitrogen atmosphere. Specimen held in a single cantilever mode was fixed at one end and the other end was vibrated by the bending stress at the frequency of $1 \mathrm{~Hz}$ with the displacement of $0.05 \mathrm{~mm}$. The glass transition temperature $\left(T_{\mathrm{g}}\right)$, storage modulus $\left(E^{\prime}\right)$ and damping properties $(\tan \delta)$ of ESO thermoset were examined with DMA. The thermal stability of ESO thermoset was characterized using TGA Pyris 6 (Perkin Elmer, USA). Approximately $5 \mathrm{mg}$ of the specimen was heated from room temperature to $600^{\circ} \mathrm{C}$ at a heating rate of $10^{\circ} \mathrm{C} / \mathrm{min}$ under nitrogen gas atmosphere.

\subsection{Water absorption of ESO thermoset}

Water absorption test according to the ASTM D570 was conducted on ESO thermoset by immersing the specimens in distilled water at room temperature for three months. The weight gained of the ESO thermoset at any time $t, M_{\mathrm{t}}$ as a result of water absorption was determined using Equation (5). The water diffusion coefficient $(D)$ of ESO thermoset was determined using Equation (6) [13]:

$$
\begin{aligned}
& M_{\mathrm{t}}=\frac{W_{\mathrm{w}}-W_{\mathrm{d}}}{W_{\mathrm{d}}} \cdot 100 \\
& \frac{M_{\mathrm{t}}}{M_{\mathrm{m}}}=1-\frac{8}{\pi^{2}} \exp \left[-\frac{D t}{h^{2}} \pi^{2}\right]
\end{aligned}
$$

where $W_{\mathrm{d}}$ and $W_{\mathrm{w}}$ represent the material dry weight and weight of materials after being exposed to the water absorption at a period of time $t$ respectively. $M_{\mathrm{m}}$ is the percentage equilibrium or the maximum water absorption of ESO thermoset, $t$ is the time taken for the ESO thermoset to reach the saturated stage, and $h$ is the thickness of the ESO specimen.

\section{Results and discussion}

\subsection{Curing characteristics of ESO thermoset}

\subsubsection{FTIR characterization}

Figure 1 shows the FTIR spectra of TEAB catalyst, MHHPA curing agent and the mixture of MHHPA/ TEAB. One may observe that FTIR spectrum of the MHHPA/TEAB mixture consists of a new $1700 \mathrm{~cm}^{-1}$ shoulder peak. The appearance of this peak is associated to the carbonyl stretch $(\mathrm{C}=\mathrm{O})$ of the aromatic acid, which indicates the asymmetric cleavage of 


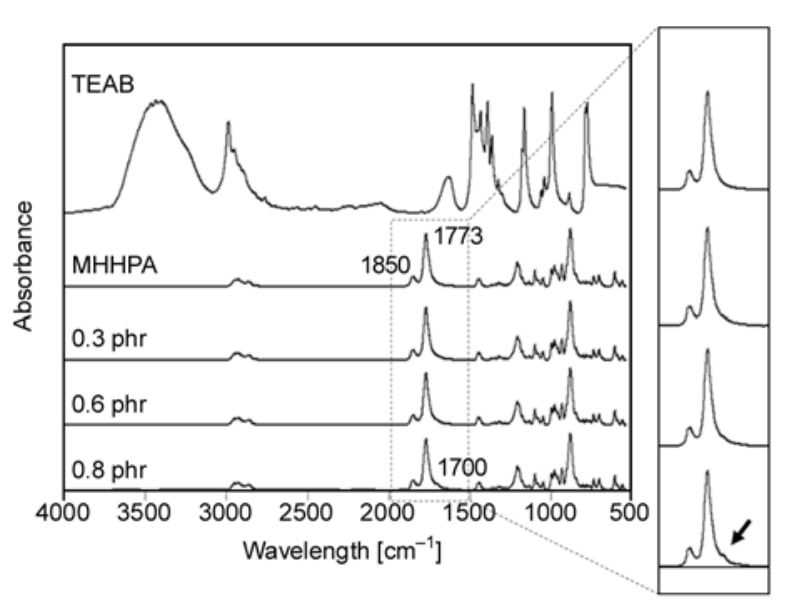

Figure 1. FTIR spectra representing the ring opening of MHHPA with TEAB (Note: Arrow shows the $1700 \mathrm{~cm}^{-1}$ shoulder peak)

the MHHPA functional group by TEAB catalyst to form the zwitterions. The amount of carbonyl groups present and the extent of MHHPA ring opening are estimated based on the carbonyl index value obtained from the FTIR study (c.f. Table 2). It is proposed that the ring opening of MHHPA curing agent by the TEAB catalyst involves $\mathrm{S}_{\mathrm{N}} 2$ reaction. The triethylamine formed as a result of the dequaternization reaction of TEAB catalyst, serves as a nucleophile and attacks the MHHPA curing agent to yield zwitterions. Although the exact absorbance band representing the zwitterions is undetected from FTIR spectra, the intensity reduction of the infrared absorbance bands that appear at 1856, 1775 and $887 \mathrm{~cm}^{-1}$ in the FTIR spectra is a good indicator to support the phenomenon of MHHPA ring opening by the TEAB catalyst. This is due to the fact that these absorbance bands are the characteristic bands for the conjugated cyclic anhydride which could be assigned to the $\mathrm{C}=\mathrm{O}$ and $\mathrm{C}-\mathrm{O}$ stretches. Considering these phenomena, a plausible assumption that

Table 2. Curing characteristics of TEAB-catalyzed ESO thermoset

\begin{tabular}{|l|c|c|c|c|c|}
\hline \multicolumn{3}{|c|}{ Characteristics } & ES_0.3A & ES_0.6A & ES_0.8A \\
\hline \multirow{4}{*}{ Carbonyl index } & $\begin{array}{l}\text { MHHPA/TEAB } \\
\text { mixture }\end{array}$ & 0.62 & 0.64 & 0.79 \\
\cline { 2 - 5 } & \multirow{2}{*}{ Curing } & $1 \mathrm{~h}$ & 1.03 & 1.69 & 1.68 \\
\cline { 2 - 5 } & $2 \mathrm{~h}$ & 2.39 & 2.96 & 3.55 \\
\cline { 2 - 5 } & $3 \mathrm{~h}$ & 2.96 & 3.79 & 3.95 \\
\hline \multicolumn{2}{|l|}{ Degree of conversion ${ }^{\mathrm{b}}[\%]$} & 95.6 & 98.6 & 99.8 \\
\hline Crosslink density $^{\mathrm{c}}\left[10^{-3} \mathrm{~mol} / \mathrm{cm}^{3}\right)$ & 0.215 & 0.260 & 0.446 \\
\hline$M_{\mathrm{c}} \mathrm{c}[\mathrm{g} / \mathrm{mol}]$ & 5116 & 4230 & 2471 \\
\hline
\end{tabular}

${ }^{\mathrm{a}}$ calculated from FTIR analysis

${ }^{\mathrm{b}}$ calculated from DSC analysis

${ }^{c}$ calculated from DMA analysis the zwitterions are generated during the pre-mixed reaction could be made.

It is noticed that the intensity of the $1700 \mathrm{~cm}^{-1}$ shoulder peak increases with increasing the TEAB catalyst concentration in MHHPA/TEAB mixture. This incident could be evidenced by the increment in the carbonyl index calculated as reported in Table 2. While, the absorbance assignable to the $\mathrm{C}=\mathrm{O}$ and $\mathrm{C}-\mathrm{O}$ stretches of MHHPA curing agent show a decreasing trend. These findings suggest the increased anhydride ring opening reaction between the TEAB catalyst and MHHPA curing agent as the TEAB catalyst concentration increases from 0.3 to $0.8 \mathrm{phr}$. This may be due to the increased potential of TEAB catalyst to facilitate the MHHPA ring opening forming more zwitterions species at higher TEAB concentration. However, it is noted that only low conversion of zwitterions is achieved during the pre-mixed reaction. The lower efficiency of the TEAB catalyst to cleavage the anhydride ring during pre-mix reaction is strongly believed to be attributed to the relatively stable and low reactivity of these quaternary ammonium salts. It is also experimentally determined in this study that no MHHPA ring opening is detected at low level of TEAB concentration $(<0.3 \mathrm{phr})$.

During the heating reaction of ESO/MHHPA/TEAB mixture at $140^{\circ} \mathrm{C}$ in the oven, the zwitterions formed would eventually react with the epoxy rings on the ESO backbone chains and subsequently generate the alkoxide intermediates. The alkoxide intermediates would then cleave another MHHPA curing agent to yield carboxylate anions during the propagation stage. These carboxylate anions would chemically react with another ESO resins to yield the reaction intermediates product and propagate the cycles. The polyesterification reaction in ESO/ MHHPA/TEAB mixture is completed after 3 hours of curing reaction. This evidently results in the formation of ESO thermoset with 3-dimensional polyester-type of linkages. To support the curing transformations proposed, the curing characteristics of ESO thermoset at three different curing times (i.e. 1, 2 and 3 hours) were evaluated using FTIR study as shown in Figure 2.

As observed in Figure 2, the intensities of absorbance bands corresponding to the $\mathrm{C}-\mathrm{O}$ and $\mathrm{C}=\mathrm{O}$ stretches of MHHPA curing agent show a progressive decrease as a function of curing reaction time for the $\mathrm{ESO} /$ 

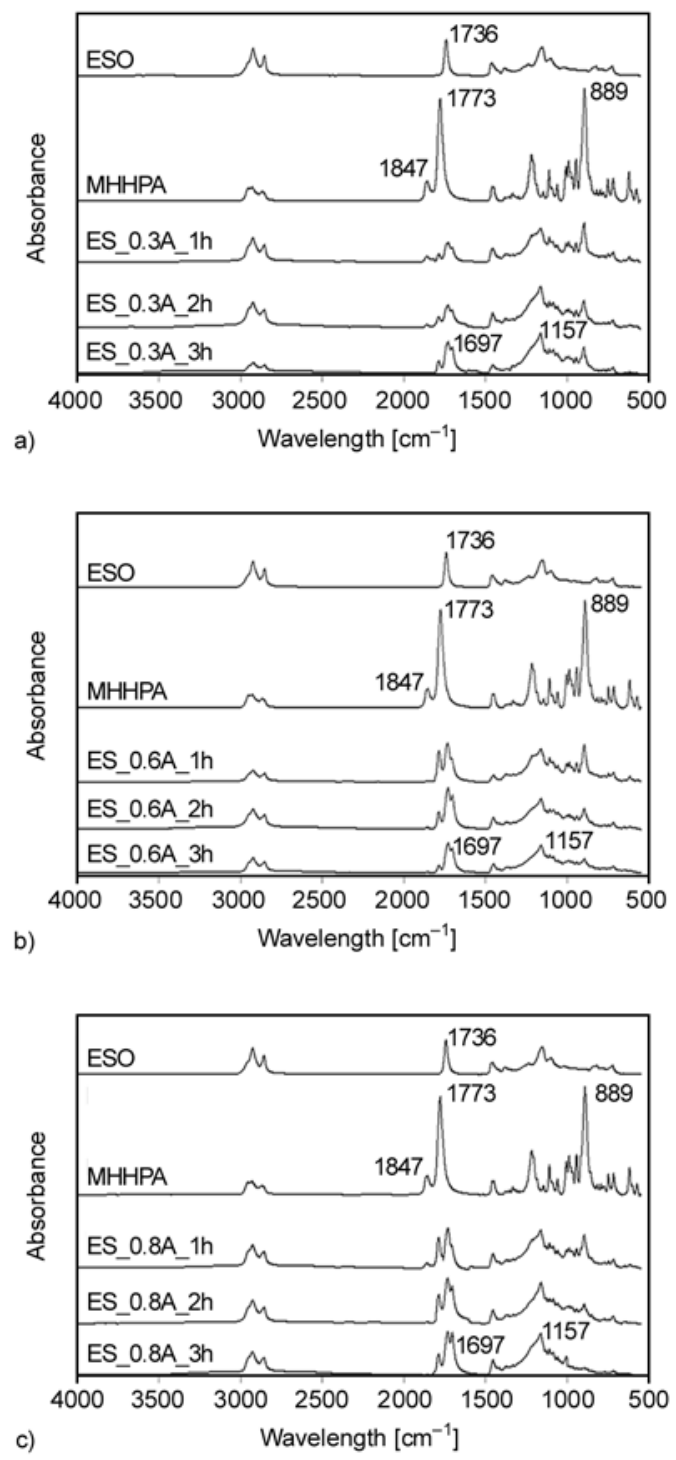

Figure 2. (a) FTIR spectra representing the curing characteristics of ES_0.3A thermoset, (b) FTIR spectra representing the curing characteristics of ES_0.6A thermoset, (c) FTIR spectra representing the curing characteristics of ES_0.8A thermoset

MHHPA/TEAB mixture. Additionally, it is clearly noticeable that the stretching vibrations of the $\mathrm{C}-\mathrm{O}-\mathrm{C}$ and $\mathrm{C}-\mathrm{C}-\mathrm{O}$ functional groups in MHHPA curing agent which in the range of $1300-1100 \mathrm{~cm}^{-1}$ disappear gradually with curing time. The reduction in intensities of these absorbance bands suggest the ring opening reaction of MHHPA curing agent and the polyesterification reaction of MHHPA curing agent with alkoxide. Furthermore, it is discovered that the absorbance bands at 1695 and $1161 \mathrm{~cm}^{-1}$ which corresponding to the $\mathrm{C}=\mathrm{O}$ and $\mathrm{O}-\mathrm{C}-\mathrm{C}$ vibrations of ester groups increasingly emerge with curing time. This finding further signifies that the poly- esterification process has taken place in the ESO/ MHHPA/TEAB mixture as these absorbance bands are the representative bands for esters. Chemical reactions of epoxy groups in ESO with carboxylate anions followed by reacting with anhydrides during the propagation cycle give rise to the formation of polyester-type linkages between ESO and MHHPA curing agents. The polyesterification reaction of ESO/MHHPA/TEAB mixture forming the polyester-type of linkages is also verified with the aid of gas chromatography-mass spectroscopy analysis (c.f. Figure 3).

From Figure 2, it is noted that the peaks which are corresponding to epoxy groups in ESO disappeared and are substituted by the peaks assignable to $\mathrm{C}=\mathrm{O}$ and $\mathrm{C}-\mathrm{O}$ functional groups of the conjugated cyclic anhydride and the $\mathrm{C}=\mathrm{O}$ and $\mathrm{O}-\mathrm{C}-\mathrm{C}$ of the ester groups after curing process for 3 hours. As shown in Figure 3, it is noted that the peak (i.e. $7.63 \mathrm{~min}$ ) corresponding to the epoxy group in ESO resin disappear after being thermally cured for 3 hours. However, the peaks (i.e. 15.81 and $20.82 \mathrm{~min}$ ) corresponding to the ester functional groups are detected in the cured ESO thermoset. Furthermore, the peak (i.e. $16.18 \mathrm{~min}$ ) representing the molecular fragments of MHHPA curing agent with ring-opened structures are also being detected in thermally cured ESO thermoset. One possible explanation to these findings could be linked to the polyesterification process of ESO/MHHPA/TEAB. Based on the information obtained from the FTIR spectra and GCMS, the curing mechanism of ESO thermoset is proposed. Figure 4 shows the proposed mechanism for the thermally cured ESO thermoset.

Furthermore, as shown in Figure 2, it is determined that the polyesterification reaction of the ESO/ MHHPA/TEAB mixture proceeds at relatively fast manner at the beginning stage during thermal curing compared to that of at the pre-mix stage. This is owing to the fact that TEAB catalyst tends to experience the chemical reaction of dequaternization at the temperature range of $100-200^{\circ} \mathrm{C}$ to generate trialkylamine. The tertiary amines formed during the internal displacement reaction will eventually boost the polyesterification rate of ESO/MHHPA/TEAB mixture. However, it is determined that the polyesterification reaction of the mixture proceeds at relatively slow manner after 2 hours of thermal curing. This event occurs as the overall catalytic reac- 

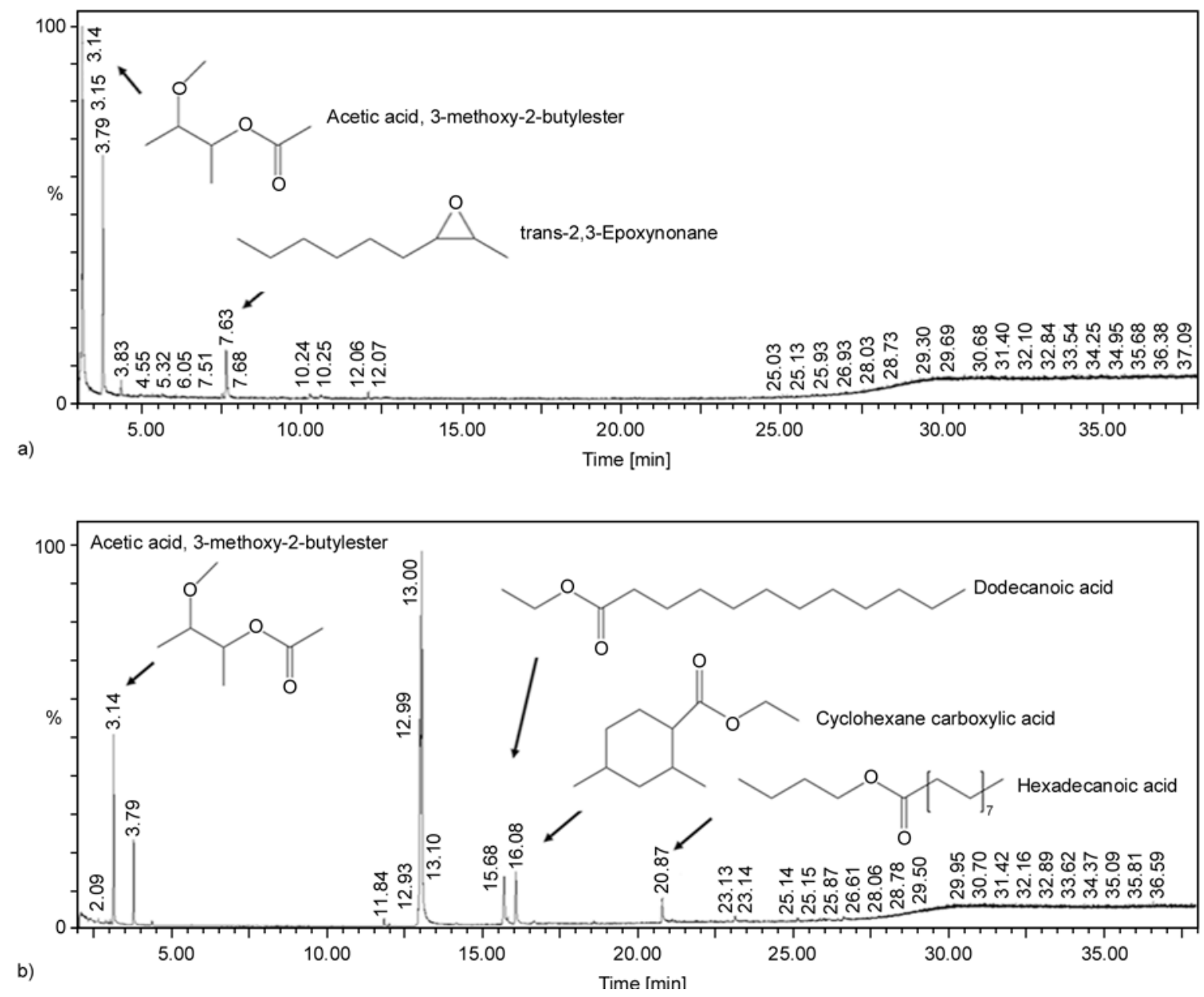

Figure 3. (a) GC-MS chromatogram of ESO, (b) GC-MS chromatogram of ES_0.6A thermoset

tion in the mixture begins to change from kinetic to diffusion-controlled. The curing process in bisphenol-S epoxy resin/phthalic anhydride epoxy system changing from kinetically controlled to diffusion controlled was also reported by Li et al. [14]. Apart from that, the effects of the TEAB concentration on the ESO thermoset are also being displayed in Figure 2. An intensity reduction in representative bands for the MHHPA curing agent and an increment in absorbance bands intensities for esters are observed when increasing the TEAB concentration from 0.3 to $0.8 \mathrm{phr}$. These results indicate the polyesterification rate is higher in thermally cured ESO containing higher TEAB concentration. Lower levels of TEAB concentration $(<0.3 \mathrm{phr})$ are also studied in this work, perhaps even a 0 -level catalyst experiment. However, it is found that the polyesterification reactions of the ESO mixture are highly unfavourable. The mixture is unable to be thermally cured at low level of TEAB concentration. One possible explanation could be linked to the very limited or even no chemical reaction between the ESO resin and MHHPA curing agent without the presence of a catalyst.

\subsubsection{DSC characterization}

The DSC heating thermograms showing the nonisothermal curing profiles of TEAB-catalyzed ESO thermoset are demonstrated in Figure 5. An exothermic peak representing the epoxy curing reactions is detected on the DSC heating curves. The enthalpy of ESO/MHHPA/TEAB polymerization can be examined by integrating the exothermic peak. It is experimentally proven that the ESO thermoset catalyzed with higher TEAB catalyst concentration shows higher $\Delta H$ value. The differences in the $\Delta H$ values obtained are mainly due to the different extent of conversion of the liquid ESO monomers to ESO thermosets during the thermal heating process in DSC. These findings show that the higher the TEAB concentration, the greater the extent of conversion in ESO thermoset, and the higher the cross- 


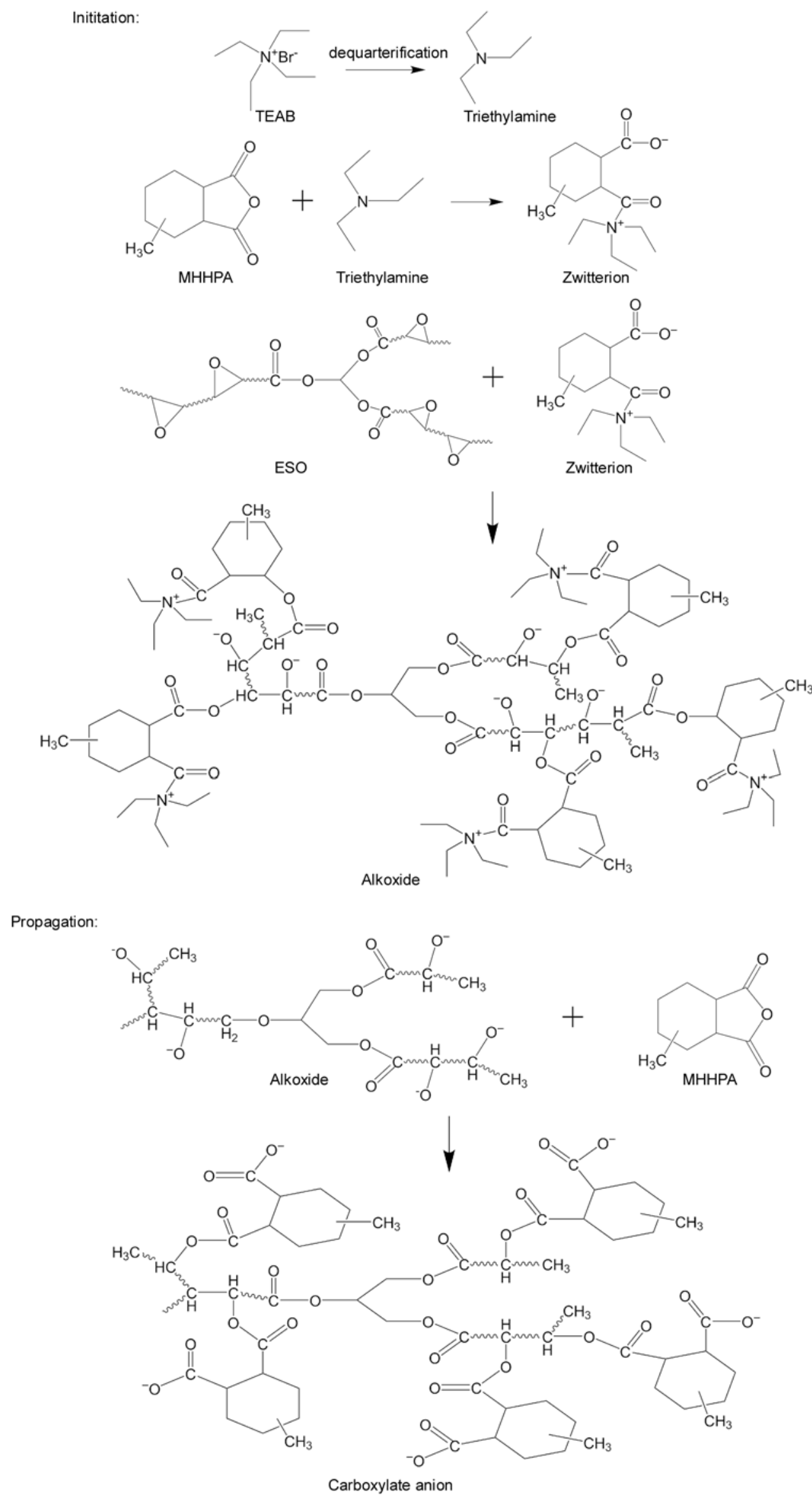

Figure 4. Curing mechanism of TEAB-catalyzed ESO thermoset 


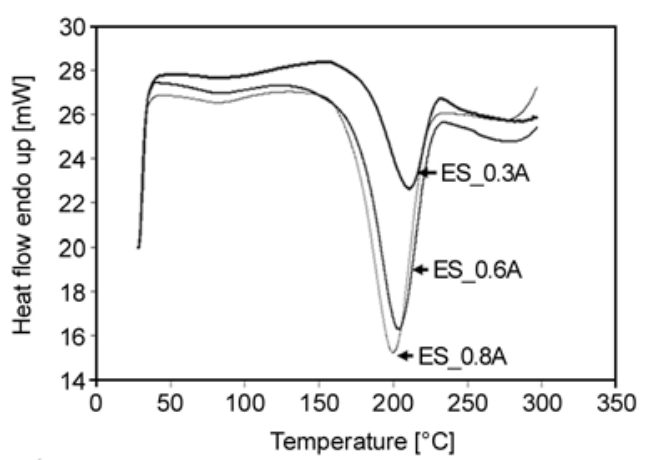

Figure 5. DSC heating thermograms of TEAB-catalyzed ESO thermoset

linking formation seems to be. One possible explanation is that the higher conversion of zwitterions is achieved during the pre-mix reaction to initiate the thermal curing reaction of ESO thermoset catalyzed with higher TEAB concentration.

From the DSC thermograms, second exotherm peak or shoulder after the $T_{\text {peak }}$ is not observable. This may give us a hint that homo-polymerizations of ESO resin do not take place. According to Boquillon and Fringant [12] who studied on the thermal curing of epoxidized linseed oil under the influence of different catalysts and anhydride hardeners, second shoulder exotherm peak after the main exotherm peak appearing at lower temperature could be associated to the epoxy homo-polymerization. Therefore, it can be concluded that ESO/MHHPA polymerization is the only chemical reaction occurs during polyesterification process and the crosslinked ESO thermoset does not contain any epoxy homopolymer. This finding is also consistent with the curing mechanism proposed (cf. Figure 4) in which the ESO resin will chemically react with the MHHPA curing agent in the presence of TEAB catalyst forming 3-dimensional polyester linkages instead of forming polyether linkages among ESO resins. It is also noticed that the $T_{\text {onset }}$ and $T_{\text {peak }}$ (c.f. Table 3 ) of the ESO thermoset shift to lower temperature as the TEAB concentration is increased from 0.3 to $0.8 \mathrm{phr}$. The shifts of DSC heating thermograms to lower temperature are presumably due to the increase in the crosslinking reaction rate. There was a direct relationship between initial curing reaction rate and catalyst concentration of a catalyzed reaction [12]. This is consistent with the finding by Liu et al. [15] which stated that the increase in catalyst concentration in epoxy-phenol/montmorillonite nanocomposites will shift $T_{\text {onset }}$ and $T_{\text {peak }}$ to lower temperature.
Table 3. Thermal properties of TEAB-catalyzed ESO thermoset

\begin{tabular}{|l|c|c|c|}
\hline \multirow{2}{*}{$\begin{array}{c}\text { Thermal } \\
\text { characteristics }\end{array}$} & \multicolumn{3}{|c|}{ ESO thermosetting resins } \\
\cline { 2 - 4 } & ES_0.3A & ES_0.6A & ES_0.8A \\
\hline$T_{\text {onset }}{ }^{\mathrm{a}}\left[{ }^{\circ} \mathrm{C}\right]$ & 183.0 & 162.1 & 150.7 \\
\hline$T_{\text {peak }}{ }^{\mathrm{a}}\left[{ }^{\circ} \mathrm{C}\right]$ & 211.9 & 203.8 & 200.8 \\
\hline$T_{\mathrm{o} 1}{ }^{\mathrm{b}}\left[{ }^{\circ} \mathrm{C}\right]$ & 200.0 & 206.0 & 210.0 \\
\hline$T_{\mathrm{o} 2}{ }^{\mathrm{b}}\left[{ }^{\circ} \mathrm{C}\right]$ & 351.0 & 342.0 & 347.0 \\
\hline$T_{\mathrm{d}} \mathrm{b}\left[{ }^{\circ} \mathrm{C}\right]$ & 458.0 & 454.0 & 456.0 \\
\hline$T_{\mathrm{g}}{ }^{\mathrm{C}}\left[{ }^{\circ} \mathrm{C}\right]$ & 53.0 & 59.0 & 69.8 \\
\hline
\end{tabular}

${ }^{a}$ detected from DSC

bdetected from TGA

${ }^{c}$ detected from DMA

Also, as shown in Figure 5, ESO thermoset undergoes the enthalpy relaxation phenomenon at relatively low TEAB concentration. The extent reduces progressively as the TEAB catalyst concentration increases from 0.3 to $0.8 \mathrm{phr}$. The reduction of this enthalpy relaxation phenomenon is believed to be due to the increase in crosslink density resulting from an increase in the extent of ESO conversion in ESO thermoset. Shin and coworkers $[16,17]$ also reported that the restriction of enthalpy relaxation can be directly related to an increase in crosslink density and activation energy for the enthalpy relaxation.

The influence of TEAB concentration on the degree of conversion $(\alpha)$ of ESO thermoset is summarized in Table 2. The degree of conversion increases about $4.2 \%$ when the TEAB concentration is increased from 0.3 to $0.8 \mathrm{phr}$. This result indicates that the efficiency of TEAB to facilitate the ring opening of MHHPA curing agent and to create more reactive sites on the anhydride (zwitterions) increases with increasing TEAB catalyst concentration. The increased number of zwitterions formation favours the polyesterification reaction in ESO thermoset and accounts for the increment in degree of conversion eventually. This finding is in good agreement with the study on the curing kinetics of bisphenol-F resin using benzyl dimethyl amine as a catalyst reported by Shokrolahi et al. [18] who determined the positive relationship between the catalyst concentration and the extent of conversion rate.

\subsubsection{DMA characterization}

The crosslink density and the molecular weight between crosslink $\left(M_{\mathrm{c}}\right)$ of the ESO thermoset as a function of TEAB concentrations are reported in Table 2. It is found that the crosslink density of 
ESO thermoset increases with TEAB concentration whereas $M_{\mathrm{c}}$ exhibits a different trend. Generally, the crosslink density and $M_{\mathrm{c}}$ obtained for the ESO thermosets are found to be lower than the epoxidized vegetable oil (EVO)/petroleum-based epoxy blends [19], but close to those triethylamine catalyzed ESO/anhydride systems [20]. The increase in TEAB concentration gives rise to an increase in the number of reactive sites on the MHHPA curing agents. The consequence is an increase in the catalyzed reaction rate and the extent of conversion. This incident will attribute to an increment in crosslink density. These findings seem to contradict with the previous findings reported by Boquillon and Fringant [12] who mentioned that the crosslink density reduces with the catalyst concentration due to the diffusional restriction of the reagent at high catalyst concentration. One possible explanation could be linked to the difference in the polymerization reaction rate in both epoxy systems. The high concentration of 2-methylimidazole (2MI) catalyst in epoxidized linseed oil (ELO)/ tetrahydrophthalic anhydride (THPA) system leads to a fast gelling of the ELO thermoset. Consequently, the diffusion of the reagents is slow and results in lower extent of conversion. While, in ESO thermoset catalyzed with low TEAB concentrations, the diffusion restriction associated to vitrification is less hindered. Therefore, the extent of conversion and crosslink density increase with the catalyst concentrations. This would inherently result in lower $M_{\mathrm{c}}$ value in ESO thermoset catalyzed with higher TEAB concentration.

\subsection{Thermal properties of ESO thermoset}

\subsubsection{Dynamic mechanical analysis}

The storage modulus $\left(E^{\prime}\right)$, damping properties $(\tan \delta)$ and the glass transition temperature $\left(T_{\mathrm{g}}\right)$ of thermally cured ESO thermoset are studied with DMA. The $E^{\prime}$ and $\tan \delta$ of cured ESO as a function of temperature are shown in Figure 6. Basically, in the testing temperature range, the ESO thermosetting resin shows three regions of viscoelasticity: the glassy state, the leathery state and the rubbery state. As shown in Figure 6, it is determined that the $E^{\prime}-T$ curve shifts to higher temperature as the TEAB concentration in the ESO thermoset increased from 0.3 to $0.8 \mathrm{phr}$. The increment in $E^{\prime}$ as a function of TEAB concentration is attributed to the increment in crosslink density and the reduction in the molec-

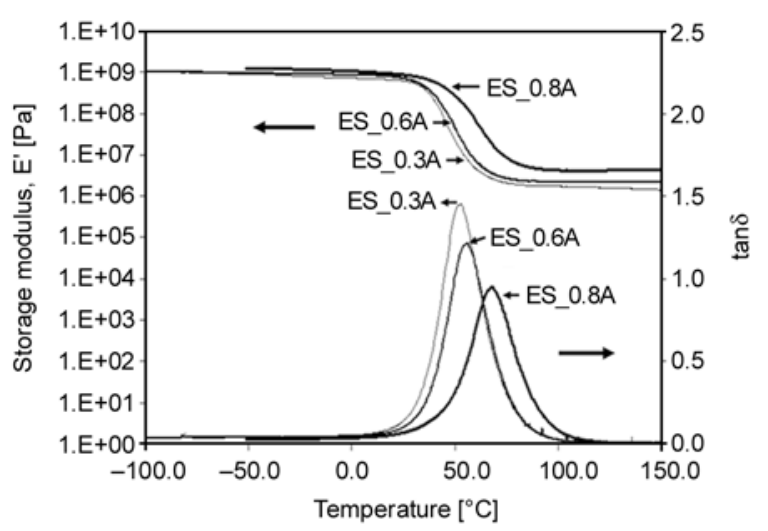

Figure 6. Effect of TEAB concentration on the $E^{\prime}$ and $\tan \delta$ of ESO thermoset

ular weight between crosslink $\left(M_{\mathrm{c}}\right)$ of the ESO thermoset. The reduction in $M_{\mathrm{c}}$ as discussed in the previous section indicates a reduction in the chain's mobility and consequently an increase in stiffness and storage modulus. This finding is in good agreement with the reduction in storage modulus with increasing $M_{\mathrm{c}}$ reported by Jin and Park [21].

Figure 6 shows the effects of TEAB concentration on the $\tan \delta$ of the ESO thermosetting materials. The shifting of $\tan \delta$ - $T$ curve to lower temperature can be associated to the depression of glass transition temperature $\left(T_{\mathrm{g}}\right)$. Damping value $(\tan \delta)$ of the cured ESO catalyzed with higher TEAB concentration is lower than that of those with lesser catalyst concentration. Higher crosslinked ESO thermosetting resin has the tendency to render higher elastic stiffness. Generally, the reduction in elastic constant will lead to the improvement in damping properties. Therefore, more flexible ESO thermosetting resins with lower crosslink densities would possess higher $\tan \delta$ peak. In addition, it can be seen that the damping peak intensity decreases with increasing TEAB concentration. The broadening and reduction in the damping peak height with increasing TEAB concentration are due to the increase in degree of crosslinking $[22,23]$. This is owing to the fact that highly cross-linked network structure can retard and restrict the chains mobility and subsequently lead to the reduction in the damping peak intensity [24].

Table 3 shows the influence of TEAB concentration on the glass transition temperature $\left(T_{\mathrm{g}}\right)$ of cured ESO thermoset. It is determined that the $T_{\mathrm{g}}$ values obtained for the TEAB-catalyzed ESO thermosets are comparable to or even higher than the existing epoxy resins based on ESO. An increment in the $T_{\mathrm{g}}$ 
value is detected if the TEAB catalyst concentration increase from 0.3 to $0.8 \mathrm{phr}$. These changes in $T_{\mathrm{g}}$ values can be related to the increase in cross-link density of the curable ESO thermoset and the reduced amounts of unreacted monomer in the ESO thermoset. The presence of unreacted monomers in the thermoset would plasticize the specimens dramatically and lower the $T_{\mathrm{g}}$ value. This is owing to the fact that the plasticizing effect of unreacted monomers will add flexibility and enhance the degree of freedom for movement of the molecular chain in the 3-dimensional crosslink network structure.

However, from the DSC characterization as discussed previously, it is determined that the degrees of conversion of TEAB-catalyzed ESO thermosets, except for the ES_0.3A thermoset, approach 99\%. Hence, the residual monomer concentration in cured ESO thermoset is found to be very low. It is therefore, expected that the observed change in $T_{\mathrm{g}}$ value is solely due to the influence of crosslink density in ESO thermoset. The increase in TEAB concentration gives rise to an increment in crosslink density and a reduction in degree of freedom for chain movements and internal rotation in the network structure. Moreover, the increase in TEAB concentration in ESO thermoset is expected to induce more complete curing reaction in which about all the unreacted monomers are built into three dimensional infusible cross-linked network structure of ESO thermoset. Thus, ESO thermoset catalyzed with higher TEAB concentration tends to possess higher $T_{\mathrm{g}}$ value.

\subsubsection{Thermogravimetry analysis}

Thermogravimetry analysis (TGA) was used in this present study to determine the thermal stability of TEAB-catalyzed ESO thermoset. Figure 7 presents the TGA and DTG curves as a function of temperature for the cured ESO thermoset. Table 3 summarizes the TGA results [i.e. onset decomposition temperature $\left(T_{\mathrm{o}}\right)$ and the maximum decomposition temperature $\left.\left(T_{\mathrm{d}}\right)\right]$ for the cured ESO thermoset. It is clearly noted in Figure 7 that ESO thermoset experiences two-stage thermal decomposition when being heated up to $600^{\circ} \mathrm{C}$. TEAB-catalyzed ESO thermoset passes through $T_{\mathrm{o} 1}$ at nearly $200^{\circ} \mathrm{C}, T_{\mathrm{o} 2}$ at $350^{\circ} \mathrm{C}$ and $T_{\mathrm{d}}$ at $450^{\circ} \mathrm{C}$. With approximation $25 \mathrm{wt} \%$ of thermal curable ESO decomposes during the first

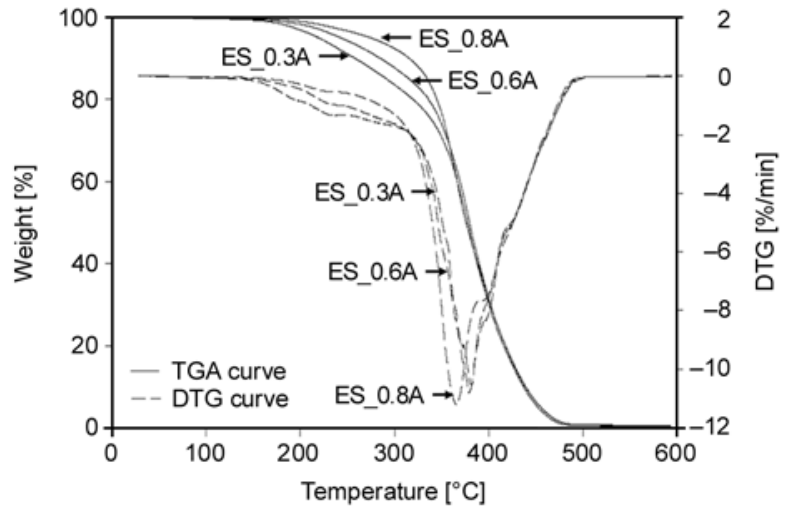

Figure 7. TGA and DTG traces of TEAB-catalyzed ESO thermosets

stage of thermal decomposition and about $71 \mathrm{wt} \%$ is lost during the second stage of decomposition. The remaining $4 \mathrm{wt} \%$ of the ESO residuals decompose totally as the heating temperature is further increased up to $500^{\circ} \mathrm{C}$.

The first stage of decomposition is believed to be due to the decomposition of the low-molecularweight components such as MHHPA curing agent or catalysts. It is believed that, generally, the lowmolecular-weight compound will show lower thermal stability as the thermal stability is determined to be proportional to molecular weight. The second stage of the major decomposition which takes place at the temperature range of 350 to $400^{\circ} \mathrm{C}$ could be attributed to the thermal decomposition of ESO component. Similar decomposition behaviour was reported by Gerbase et al. [20] for the ESO/triethylamine/dodecenylsuccinic anhydride mixtures, where two different thermal decomposition stages present. Also, Hazer et al. [25] found that the decomposition temperature of the soya components in microbial elastomers is at around $310^{\circ} \mathrm{C}$. Referring to Figure 7, the first stage effect of ESO thermoset with different TEAB concentration is found to be diverged from each other. ESO thermoset with higher TEAB concentration is determined to be more thermal stable than those thermosets with lower concentration. This finding is believed to be attributed to the increase in degree of crosslinking and crosslink density as the TEAB catalyst concentration is increased. Foussier and Rabek [26] also reported in their study that polymer system with highly cross-linked network structure commonly exhibit greater thermal stability than those systems with lower crosslinking. 


\subsection{Kinetics of water absorption of ESO thermoset}

Figure 8 shows the effect of TEAB concentration on the water uptake of the ESO thermoset after subjected to water absorption test. It is seen that the water uptake of all TEAB-catalyzed ESO thermosetting resins reach the saturation stage at approximately 2000 hours. This is owing to the fact that the water uptake of TEAB-catalyzed ESO thermoset has achieved the equilibrium condition. The water uptake would reach the real saturation at equilibrium stage, in which all the micro-voids in the 3dimensional crosslinked network structure are filled with water molecules [27]. Water uptake of cured ESO thermosets is attributed to the presence of free volume and hydrophilic functional groups such as hydroxyl groups in their backbone structure. The hydroxyl groups in cured ESO thermoset tend to attract water molecules which are polar in nature. The water molecules absorbed in ESO thermoset may exist as bound water or unbounded cluster.

It is clearly noted that the maximum water uptake $\left(M_{\mathrm{m}}\right)$ and water diffusion coefficient $(D)$ of the cured TEAB-catalyzed ESO thermoset decrease with increasing TEAB concentration as shown in Table 4. It is determined that increase the number of crosslink in cured ESO thermoset would reduce the penetration of water molecules into the ESO network structure. This is owing to the fact that the intense crosslink network would hinder the water molecules to diffuse into the free volume followed by residing in the volume available in the ESO thermoset. Research study conducted by Saijun et al., [28] also showed that polymeric systems with higher crosslink density will possess lower free volume and lower water uptake. The water absorption

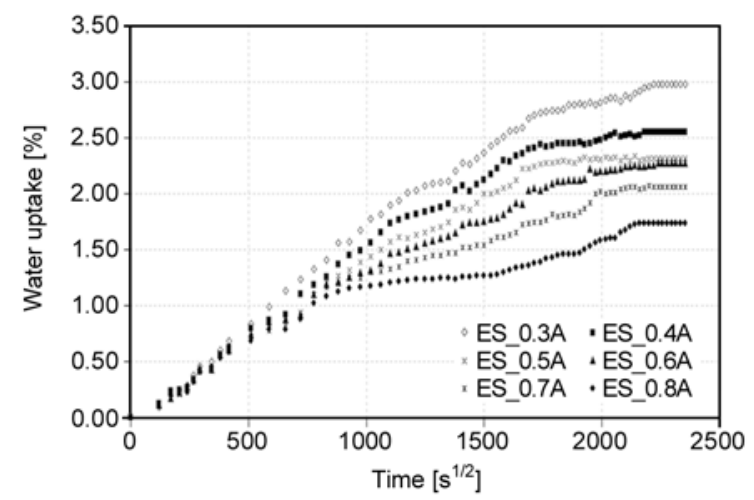

Figure 8. Effect of TEAB concentration on water uptake of ESO thermoset
Table 4. Maximum water absorption $\left(M_{\mathrm{m}}\right)$ and diffusion coefficient $(D)$ of ESO thermoset

\begin{tabular}{|c|c|c|c|}
\hline \multicolumn{2}{|c|}{ ESO thermoset } & $\mathbf{M}_{\mathbf{m}}[\mathbf{\%}]$ & $\mathbf{D}\left[\mathbf{1 0}^{\mathbf{- 1 2}} \mathbf{~ m}^{\mathbf{2}} \mathbf{/ s}\right]$ \\
\hline \multirow{4}{*}{ TEAB-catalyzed } & ES_0.3A & 2.94 & 1.09 \\
\cline { 2 - 4 } & ES_0.4A & 2.60 & 0.87 \\
\cline { 2 - 4 } & ES_0.5A & 2.34 & 0.73 \\
\cline { 2 - 4 } & ES_0.6A & 2.28 & 0.70 \\
\cline { 2 - 4 } & ES_0.7A & 2.09 & 0.51 \\
\cline { 2 - 4 } & ES_0.8A & 1.77 & 0.48 \\
\hline
\end{tabular}

behaviour is highly dependent on the free volume and chemical nature of polymer material. The high TEAB concentration in ESO thermoset would give rise to an increase in crosslinking and a decrease in free volume. This phenomenon will eventually lead to a reduction in the capability of the water molecules to penetrate and reside in the free volume. Therefore, the maximum water uptake and water diffusion coefficient of the ESO thermoset decrease with increasing TEAB concentration.

\section{Conclusions}

Based on the work devoted to study the effect of tetraethylammonium bromide concentration on the curing characteristic, thermal properties and kinetics of water absorption of ESO thermoset, the following conclusions can be drawn:

The polyesterification rate, degree of conversion and crosslink density were higher for ESO thermoset catalyzed with higher TEAB concentration. From the DMA and TGA studies, it was found that ESO thermoset with higher TEAB concentration possessed higher storage modulus, glass transition temperature and thermal stability. It was found that the water absorption behaviour of ESO thermoset obeyed Fickian law. The maximum water absorption $\left(M_{\mathrm{m}}\right)$ and water diffusion coefficient $(D)$ of the ESO thermosets were in the range of 1.77 to $2.94 \%$ and 0.48 to $1.09 \cdot 10^{-12} \mathrm{~m}^{2} / \mathrm{s}$ respectively. Water uptake and diffusivity of ESO thermoset can be reduced by increasing the crosslink density in the thermoset.

\section{Acknowledgements}

Authors would like to express their appreciation to Universiti Sains Malaysia for the Incentive Grant (8021013) and the Research University Postgraduate Research Grant Scheme (USM-RU-PRGS 8043019). Authors also thank Ministry of Science, Technology and Innovation (MOSTI), Malaysia for the National Science Foundation fellowship. 


\section{References}

[1] Keles E., Hazer B.: Synthesis of segmented polyurethane based on polymeric soybean oil polyol and poly (ethylene glycol). Journal of Polymers and the Environment, 17, 153-158 (2009).

DOI: $10.1007 / \mathrm{s} 10924-009-0132-0$

[2] Petrović S., Xu Y. J., Milić J., Glenn G., Klamczynski A.: Biodegradation of thermoplastic polyurethanes from vegetable oils. Journal of Polymers and the Environment, 18, 94-97 (2010).

DOI: $10.1007 / \mathrm{s} 10924-010-0194-\mathrm{Z}$

[3] Cakmakli B., Hazer B., Acikgöz S., Can M., Cömert B.: PMMA-multigraft copolymers derived from linseed oil, soybean oil, and linoleic acid: Protein adsorption and bacterial adherence. Journal of Applied Polymer Science, 105, 3448-3457 (2007).

DOI: $10.1002 / a p p .26397$

[4] Xia Y., Henna H., Larock C.: Novel thermosets from the cationic copolymerization of modified linseed oils and dicyclopentadiene. Macromolecular Materials and Engineering, 294, 590-598 (2009).

DOI: $10.1002 /$ mame.200900060

[5] Andjelkovic D., Lu Y. S., Kessler R., Larock C.: Novel rubbers from the cationic copolymerization of soybean oils and dicyclopentadiene, 2 - Mechanical and damping properties. Macromolecular Materials and Engineering, 294, 472-483 (2009).

DOI: $10.1002 /$ mame.200900053

[6] Liu Z., Erhan S. Z.: Ring-opening polymerization of epoxidized soybean oil. Journal of the American Oil Chemists' Society, 87, 437-444 (2010).

DOI: $10.1007 / \mathrm{s} 11746-009-1514-0$

[7] Jin F-L., Park S-J.: Thermal and rheological properties of vegetable oil-based epoxy resins cured with thermal latent initiator. Journal of Industrial and Engineering Chemistry, 13, 808-814 (2007).

[8] Gupta A. P., Ahmad S., Dev A.: Development of novel bio-based soybean oil epoxy resins as a function of hardener stoichiometry. Polymer-Plastics Technology and Engineering, 49, 657-661 (2010).

DOI: $\underline{10.1080 / 03602551003681796}$

[9] Ortiz R. A., López D. P., Cinneros M. L. G., Valverde J. C. R., Crivello V.: A kinetic study of the acceleration effect of substituted benzyl alcohols on the cationic photopolymerization rate of epoxidized natural oils. Polymer, 46, 1535-1541 (2005).

DOI: $10.1016 /$ j.polymer.2004.12.020

[10] Gao Y.: Synthesis and evaluation of vegetable (soybean) oil based epoxy resin for beverage can coating. $\mathrm{PhD}$ thesis, Missouri Universiti of Science and Technology (2002).

[11] Tan S. G., Chow W. S.: Thermal properties, fracture toughness and water absorption of epoxy-palm oil blends. Polymer-Plastics Technology and Engineering, 49, 900-907 (2010).

DOI: $10.1080 / 03602551003682042$
[12] Boquillon N., Fringant C.: Polymer networks derived from curing of epoxidised linseed oil: Influence of different catalysts and anhydride hardeners. Polymer, 41, 8603-8613 (2000).

DOI: 10.1016/S0032-3861(00)00256-1

[13] Tham W. L., Chow W. S., Mohd Ishak Z. A.: Simulated body fluid and water absorption effects on poly(methyl methacrylate)/hydroxyapatite denture base composites. Express Polymer Letters, 4, 517-528 (2010).

DOI: $10.3144 /$ expresspolymlett.2010.66

[14] Li Y., Gao J., Liu G., Zhang R.: Cure kinetics and thermal property of bisphenol-S epoxy resin and phthalic anhydride. International Journal of Polymeric Materials, 49, 441-455 (2001).

DOI: $10.1080 / 00914030108035876$

[15] Liu D., Shi Z., Matsunaga M., Yin J.: DSC investigation of the hindered effect on curing behavior for epoxy-phenol/MMT nanocomposites based on the acidic octadecylamine modifier. Polymer, 47, 29182927 (2006).

DOI: $10.1016 /$ j.polymer.2006.02.010

[16] Shin J., Nazarenko S., Hoyle E.: Effects of chemical modification of thiol-ene networks on enthalpy relaxation. Macromolecules, 42, 6549-6557 (2009).

DOI: $10.1021 / \mathrm{ma9001403}$

[17] Shin J., Nazarenko S., Paige Phillips J., Hoyle E.: Physical and chemical modifications of thiol-ene networks to control activation energy of enthalpy relaxation. Polymer, 50, 6281-6286 (2009). DOI: $10.1016 /$ j.polymer.2009.10.053

[18] Shokrolashi F., Sadi M., Shokrolahi P.: A study on curing kinetic of bisphenol-F using benzyl dimethyl amine by isothermal DSC. Journal of Thermal Analysis and Calorimetry, 82, 151-156 (2005). DOI: $10.1007 / \mathrm{s} 10973-005-0856-6$

[19] Tan S. G., Chow W. S.: Biobased epoxidized vegetable oils and its greener epoxy blends: A review. PolymerPlastics Technology and Engineering, 49, 1581-1590 (2010).

DOI: $10.1080 / 03602559.2010 .512338$

[20] Gerbase A. E., Petzhold C. L., Costa P. O.: Dynamic mechanical and thermal behavior of epoxy resins based on soybean oil. Journal of the American Oil Chemists' Society, 79, 797-802 (2002). DOI: 10.1007/s11746-002-0561-z

[21] Jin F-L., Park S-J.: Impact-strength improvement of epoxy resins reinforced with a biodegradable polymer. Materials Science and Engineering: A, 478, 402-405 (2008).

DOI: $10.1016 /$ j.msea.2007.05.053

[22] Banik I., Bhowmick K.: Dynamic mechanical properties of electron beam modified fluorocarbon rubber. Journal of Applied Polymer Science, 69, 2079-2087 (1998).

DOI: 10.1002/(SICI)1097-4628(19980906)69:10<2079 ::AID-APP19>3.0.CO;2-X 
[23] Jacobs P. M., Jones F. R.: The influence of heterogeneous crosslink density on the thermomechanical and hygrothermal properties of an unsaturated polyester resin: 1. Thermomechanical response. Polymer, 34, 2122-2127 (1993).

DOI: 10.1016/0032-3861(92)90116-E

[24] Rials G., Glasser G.: Engineering plastics from lignin IV. Effect of crosslink density on polyurethane film properties - Variation in $\mathrm{NCO}: \mathrm{OH}$ ratio. International Journal of the Biology, Chemistry, Physics and Technology of Wood, 38, 191-199 (1984).

DOI: $10.1515 / \mathrm{hfsg} .1984 .38 .4 .191$

[25] Hazer B., Hazer D. B., Coban B.: Synthesis of microbial elastomers based on soybean oil. Autoxidation kinetics, thermal and mechanical properties. Journal of Polymer Research, 17, 567-577 (2010).

DOI: $10.1007 / \mathrm{s} 10965-009-9345-0$
[26] Fouassier J. P., Rabek J. F.: Radiation curing in polymer science and technology: Fundamentals and methods. Elsevier, London (1993).

[27] Chow W. S.: Water absorption of epoxy/glass fiber/ organo-montmorillonite nanocomposites. Express Polymer Letters, 1, 104-108 (2007).

DOI: $10.3144 /$ expresspolymlett.2007.18

[28] Saijun D., Nakason C., Kaesaman A., Klinpituksa P.: Water absorption and mechanical properties of waterswellable natural rubber. Songklanakarin Journal of Science and Technology, 31, 561-565 (2009). 